\title{
Change-point of Multiple Biomarkers in Women with Ovarian Cancer
}

Inés P. Mariño ${ }^{1 *}, 2 \mathbb{I}$, Oleg Blyuss ${ }^{2}, \mathbb{I}$, Andy Ryan $^{2}$, Aleksandra Gentry-Maharaj ${ }^{2}$, John F. Timms², Anne Dawnay $^{3}$, Jatinderpal Kalsi², Ian Jacobs ${ }^{2,4}$, Usha Menon ${ }^{2, \&}$, Alexey Zaikin ${ }^{2, \&}$

1 Departamento de Biología y Geología, Física y Química Inorgánica, Universidad Rey Juan Carlos, Móstoles, Madrid 28933, Spain.

2 Department of Women's Cancer, Institute for Women's Health, University College London, London WC1E 6BT, United Kingdom.

3 Clinical Biochemistry, University College London Hospitals, London W1T 4EU, United Kingdom.

4 University of New South Wales, Sydney, NSW 2052, Australia.

IThese authors contributed equally to this work.

\& These authors also contributed equally to this work.

* ines.perez@urjc.es

\section{Abstract}

To date several algorithms for longitudinal analysis of ovarian cancer biomarkers have been proposed in the literature. An issue of specific interest is to determine whether the baseline level of a biomarker changes significantly at some time instant (change-point) prior to the clinical diagnosis of cancer. Such change-points in the serum biomarker Cancer Antigen 125 (CA125) time series data have been used in ovarian cancer screening, resulting in earlier detection with a sensitivity of $85 \%$ in the most recent trial, the UK Collaborative Trial of Ovarian Cancer Screening (UKCTOCS, number ISRCTN22488978; NCT00058032). Here we propose to apply a hierarchical Bayesian change-point model to jointly study the features of time series from multiple biomarkers. For this model we have analytically derived the conditional probability distribution of every unknown parameter, thus enabling the design of efficient Markov chain Monte Carlo methods for their estimation. We have applied these methods to the estimation of change-points in time series data of multiple biomarkers, including CA125 and others, using data from a nested case-control study of women diagnosed with ovarian cancer in UKCTOCS. In this way we assess 
whether any of these additional biomarkers can play a role in change-point detection and, therefore, aid in the diagnosis of the disease in patients for whom the CA125 time series does not display a change-point. We have also investigated whether the change-points for different biomarkers occur at similar times for the same patient. The main conclusion of our study is that the combined analysis of a group of specific biomarkers may possibly improve the detection of change-points in time series data (compared to the analysis of CA125 alone) which, in turn, are relevant for the early diagnosis of ovarian cancer.

\section{Introduction}

Cancer of the ovary is the fifth most common cause of cancer-related deaths among women, with more than 140,000 deaths worldwide each year. Most cases occur in post-menopausal women (75\%), with an incidence of 40 per 100,000 per year in women aged over 50. Diagnosis of ovarian cancer at Stage I, when the tumour is confined to the ovary, results in a 5-year survival of $90 \%$ [1. However, the 5 -year survival decreases sharply when cancer diagnosis occurs at later stages such as Stage III (20\%) and Stage IV (3\%). This suggests that the development of new approaches for longitudinal multi-marker analysis that result in earlier detection of ovarian cancer may significantly impact on mortality 24.

One of the most successful methods of detection of ovarian cancer in a screening context to date is the statistical inference technique for the longitudinal analysis of ovarian cancer biomarkers developed by Skates et al. [5] 7], where the main assumption is the existence of a change-point in the serum CA125 time series as the tumour develops. In particular, the level of CA125 is assumed to remain approximately constant until, at some time instant, it begins to increase significantly. The latter time point is referred to as a change-point. The algorithm proposed in 5 is based on a hierarchical Bayesian model that includes the change-point as one of the random parameters to be estimated.

In this paper, we jointly analyse time series data from multiple biomarkers to determine whether the level of these markers changes significantly and coherently at specific time instants. The detection of such a change-point may contribute to the earlier diagnosis of the disease. Although the serum CA125 is the most useful biomarker in the screening of ovarian cancer, multiple serum biomarkers have been reported to be associated with the development of ovarian cancer and to possibly improve the performance of CA125 when used in combination [8 17]. The biomarker that has received more attention is the Human Epididymis Protein 4 (HE4), which has been used in the ROMA (Risk of Ovarian Malignancy 
Algorithm) to discriminate ovarian cancer from benign diseases 8, 18, as well as in different panels for the ${ }^{23}$ purpose of early detection 9 11. In a study within the Prostate Lung Colorectal and Ovarian (PLCO) ${ }_{24}$ cancer screening trial [19, HE4 was the second best marker after CA125 with a sensitivity of $73 \%$ (95\% 25 confidence interval $0.60-0.86$ ) compared to $86 \%$ (95\% confidence interval $0.76-0.97)$ for CA125 [12 20. ${ }_{26}^{26}$ Another serum biomarker glycodelin has also shown promising performance in the detection of ovarian ${ }_{27}$ cancer $13,14,21$. Other markers that appear to be promising when used in multi-marker panels include ${ }_{28}$ matrix metalloproteinase-7 (MMP7) [13, 20, 22], cytokeratin 19 fragment (CYFRA 21-1) [15, 20] and 29 mesothelin (MSLN) [11,16].

In order to incorporate this information we assume a hierarchical Bayesian change-point model for different biomarkers in addition to serum CA125. Statistical inference in this model can be carried out using Markov chain Monte Carlo (MCMC) methods 23. In particular, we have analytically obtained the full conditional probability distributions for all the unknown parameters in the model, thus enabling the design of an efficient Metropolis-within-Gibbs algorithm 24 for their Bayesian estimation. We apply this technique to the estimation of change-points in time series data, including CA125 and the other biomarkers in patients diagnosed with ovarian cancer and in a control group of healthy individuals. We assess whether any of these additional biomarkers can play a role in ovarian cancer diagnosis by either detecting a change-point in any of the available biomarkers earlier than in CA125 or by detecting a change-point in women in whom the CA125 does not display a change-point. We also investigate whether the change-points for different biomarkers occur at similar time points.

The Bayesian estimation approach advocated in this paper aims at producing a full statistical ${ }_{42}$ characterisation of the unknown model parameters, given in the form of their posterior probability ${ }_{43}$ distribution conditional on the available data. The proposed Metropolis-within-Gibbs algorithm yields a ${ }_{44}$ Monte Carlo approximation of this posterior distribution, which enables the implementation of a variety ${ }_{45}$ of estimators for the parameters and provides the means to evaluate their accuracy and reliability as well. $\quad 46$ While in this paper we keep the change-point estimation process relatively simple, the framework and ${ }_{47}$ algorithms described in Sections 2 and 3 lends itself to potentially advantageous extensions.

The rest of this paper is organised as follows. Section 2 is devoted to the description of the dataset 49 and the hierarchical Bayesian model used to represent it. The inference algorithm for the detection and 50 estimation of change-points in biomarker time series is introduced in Section 3 . The results obtained for 51 the available dataset are shown and discussed in Section 4 Section 5 is devoted to the conclusions. 


\section{Data model}

\section{$2.1 \quad$ Dataset}

In this study we have used a dataset from the multimodal arm of the UK Collaborative Trial of Ovarian Cancer Screening (UKCTOCS) 25, where women underwent annual screening using the blood tumour marker CA125. HE4, MMP7, CYFRA_21-1, Glycodelin and MSLN assays were performed on stored serial samples from a subset of women in the multimodal arm diagnosed with ovarian cancer. The dataset included 179 controls (healthy subjects) and 44 cases (diagnosed patients): 35 invasive epithelial ovarian cancer (iEOC), 3 fallopian tube cancer and 6 peritoneal cancer. Out of these 44 cases, 16 are early stage (FIGO 26 stages I and II) and 28 are late stage (FIGO stages III and IV). In terms of histology, there are 27 serous cancers, 2 papillary, 3 endometrioid, 2 clear cell, 3 carcinosarcoma, and 7 not specified cancers. Each control has 4 to 5 serial samples available (177 controls with 5 samples and 2 controls with 4 samples) and each case has 2 to 5 serial samples available (24 cases with 5 samples, 10 cases with 3 samples and 10 cases with 2 samples). The range of ages for the healthy subjects (controls) is 50.3-78.8 years and the average age over all the subjects and samples is 63.6 years. The range of ages for the diagnosed patients (cases) is 52-77.4 years and the average age over all patients and samples is 65.5 years.

It should be noted here that all the biomarker measurements have been modified via a logarithmic transformation, as detailed in 5, 14, in the form of $Y=\log (Z+4)$, where $Z$ is the value of a particular marker. For most patients with ovarian cancer prior to disease diagnosis, serum CA125 rises exponentially. This transformation allows us to observe a linear change in time.

\section{$2.2 \quad$ Model}

Figure 1 shows the scheme of the hierarchical Bayesian model for patients diagnosed with ovarian cancer. Let $Y_{i j}$ denote the log-transformed measurement of the biomarker $Z$ (where $Z$ can be any of CA125, ${ }^{74}$ HE4, Glycodelin, MSLN, MMP7 or CYFRA_21-1) for the $i$-th patient in the study at age $t_{i j}$, where 75 $j=1, \ldots, k_{i}$ represents the ordinal of the observation for patient $i$ (i.e., the first observation, the second 76 observation, and so on), being $k_{i}$ the total number of measurements for patient $i$. The values of the $\quad 77$ biomarkers are collected at time points $t_{i j}$, which can depend on previous values $Y_{i j^{\prime}}, j^{\prime}<j$. For this ${ }_{78}$ model, an unobserved binary indicator $I_{i}$ is included to distinguish subjects whose ovarian cancer does $\quad 79$ $\left(I_{i}=1\right)$, or does not $\left(I_{i}=0\right)$, produce an increased biomarker level. Notice that for patients with $I_{i}=0$ 
there is no way to tell them from healthy individuals by looking at that biomarker alone. Within the ${ }_{81}$ proposed hierarchical model, separation of cases that do not produce a change-point from those that ${ }_{82}$ do produce it enables a more precise estimation of individual change-points and rates of change. The ${ }_{83}$ indicator $I_{i}$ for each case is assumed to follow, a priori, a Bernoulli distribution with success probability ${ }^{84}$ $\pi$, where $\pi$ represents the proportion of cases that produce an increased biomarker level. As in [5], in 85 light of the observation of Kabawat et al. [27] that approximately $15 \%$ of ovarian cancer cases do not 86 produce an excess of biomarker CA125, a Beta(42.5,7.5) prior distribution with mean 0.85 and standard 87 deviation 0.05 is adopted as the prior distribution of $\pi$. We assume the same prior distribution of $\pi$ for all $\quad 88$ the biomarkers studied in this paper.

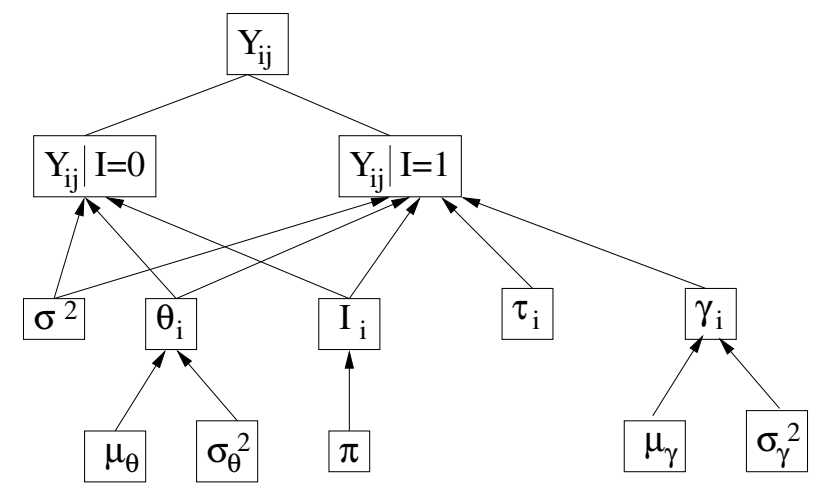

Fig 1. Scheme of the hierarchical model for women with cancer.

When the binary indicator is null, $I_{i}=0$, the transformed marker level $Y_{i j}$ is assumed to have a constant mean, denoted $E\left(Y_{i j} \mid t_{i j}, I_{i}=0\right)=\theta_{i}$. Because the biomarkers are measured with some error, 91 variations around the expected level are modelled by a normal distribution with zero mean and variance 92 $\sigma^{2}$. Thus, the marker level is conditionally distributed as $Y_{i j} \mid\left\{I_{i}=0\right\} \sim \mathcal{N}\left(\theta_{i}, \sigma^{2}\right)$, where $\mathcal{N}(a, b)$ denotes $\quad 93$ a normal distribution with mean $a$ and variance $b$. For $\sigma^{2}$, we have chosen an inverse gamma distribution, $\quad 94$ namely $\sigma^{2} \sim I G(2.05,0.1)$ (where $I G(a, b)$ denotes the inverse gamma distribution with mean $b /(a-1) \quad 95$ and variance $\left.b^{2} /\left[(a-1)^{2}(a-2)\right]\right)$, instead of the improper Jeffreys' prior used in [5]. We have specifically 96 selected prior distributions which are flexible (note the large variances of the IG distributions of choice, 97 which makes them hardly informative) yet proper 1 . An independent normal distribution is a priori 98 assumed for the individual's mean biomarker level, thus $\theta_{i} \sim \mathcal{N}\left(\mu_{\theta}, \sigma_{\theta}^{2}\right)$, where we have chosen the same 99

\footnotetext{
${ }^{1}$ The selection of proper priors is important. Under improper priors, such as the Jeffrey's priors utilised in [5], it becomes necessary to verify whether the posterior distributions are proper or not. If they are not, any computational inference methods applied to approximate the posterior distributions may become numerically unstable and yield unreliable estimates of the quantities of interest.
} 
priors as in 5 for $\mu_{\theta}$ and $\sigma_{\theta}$, that is, $\mu_{\theta} \sim \mathcal{N}(2.75,1)$ and $\sigma_{\theta}^{2} \sim I G(2.04,0.065)$.

When the binary indicator is $I_{i}=1$, the log-transformed biomarker level starts increasing at a 101 positive rate $\gamma_{i}$ after the unobserved time instant $\tau_{i}$, which is modelled as random and referred to as the ${ }_{102}$ change-point of the time series. As a consequence, the mean of the biomarker level is represented by way ${ }_{103}$ of the piecewise linear function

$$
E\left(Y_{i j} \mid t_{i j}, I_{i}=1\right)=\theta_{i}+\gamma_{i}\left(t_{i j}-\tau_{i}\right)^{+}
$$

where $(\cdot)^{+}$denotes the positive part of the expression between brackets and $\theta_{i}$ is random and modelled ${ }_{105}$ in the same way as described above. A normal distribution with constant variance $\sigma^{2}$ is again specified ${ }_{106}$ to model variations around the expected level. Thus, the marker level is conditionally distributed as ${ }_{107}$ $Y_{i j} \mid\left\{I_{i}=1\right\} \sim \mathcal{N}\left(\theta_{i}+\gamma_{i}\left(t_{i j}-\tau_{i}\right)^{+}, \sigma^{2}\right)$, where the prior for $\sigma^{2}$ has been chosen the same as before, i.e., ${ }_{108}$ $\sigma^{2} \sim I G(2.05,0.1)$. Independent normal distributions are assumed for the individual random effects, 109 thus $\log \gamma_{i} \sim \mathcal{N}\left(\mu_{\gamma}, \sigma_{\gamma}^{2}\right)$, where $\mu_{\gamma} \sim \mathcal{N}(1.1,0.1)$ as in $\left[5\right.$ and the prior for $\sigma_{\gamma}^{2}$ has been chosen as ${ }_{110}$ $\sigma_{\gamma}^{2} \sim I G(2.2,0.12)$. Finally, $d_{i}$ denotes the age of patient $i$ at the time of the last measurement, i.e. the age ${ }_{111}$ at the time of clinical detection of the disease. The distribution of the preclinical duration $\delta_{i}=d_{i}-\tau_{i}$ in ${ }_{112}$ years has been estimated from the ratio of prevalence to incidence in a series of screening trials $[28] 30$ to ${ }_{113}$ be normal with mean 2.0 and standard error 0.75 , truncated to the interval $[0,5]$. This yields independent ${ }_{114}$ truncated normal models for each change-point, $\tau_{i}$, with mean age $d_{i}-2.0$ and standard deviation $0.75, \quad{ }_{115}$ truncated to the interval $\left[d_{i}-5, d_{i}\right]$, denoted $\mathcal{T N}\left(d_{i}-2,0.75^{2}, d_{i}-5, d_{i}\right)$.

\section{Inference algorithm}

\subsection{Metropolis-within-Gibbs sampling}

We propose to compute Bayesian estimates of the unknown parameters in the model using a Gibbs ${ }_{119}$ sampling scheme [23]. To implement this kind of method, it is necessary to obtain the full conditional ${ }_{120}$ distributions of all the parameters in the model. These distributions have been explicitly derived and are ${ }_{121}$ given in Appendix $\mathrm{A}$, together with the joint density of all the parameters and the observed data, being one ${ }_{122}$ of the contributions of this work. Notice that the conditional distributions of $\tau_{i}$ and $\log \gamma_{i}$ are not standard ${ }_{123}$ and therefore it is not possible to draw samples from them directly. To circumvent this difficulty, we run ${ }^{124}$ 
several steps of a Metropolis-Hastings (MH) algorithm for each one of the parameters $\tau_{i}$ and $\log \gamma_{i}$ (and ${ }^{125}$ for each patient $i$ ) at each iteration of the Gibbs sampler (see Section 3.1). The resulting sampling scheme ${ }_{126}$ is often termed Metropolis-within-Gibbs $(\mathrm{MwG})$ 24] or Component-wise Metropolis-Hastings 31 33. The ${ }^{127}$ need to generate a relatively long chain of samples in the embedded $\mathrm{MH}$ algorithms has been discussed in ${ }_{128}$ the literature 41 43]. In this paper, we generate a chain of 200 samples for each of the variables $\tau_{i}$ and ${ }^{129}$ $\log \gamma_{i}$ at each iteration of the MwG algorithm.

The proposed MwG algorithm iteratively generates samples from the distribution of each parameter ${ }_{131}$ conditional on the current values of the other parameters. It can be shown that the resulting sequence of ${ }_{132}$ samples yields a Markov chain, and the stationary distribution of that Markov chain is the joint posterior ${ }_{133}$ probability distribution 24].

In order to describe the proposed $\mathrm{MwG}$ sampling algorithm let us introduce the sets

$$
C=\left\{\mu_{\theta}, \mu_{\gamma}, \sigma_{\theta}^{2}, \sigma_{\gamma}^{2}, \sigma^{2}, \pi\right\}=\left\{c_{1}, c_{2}, c_{3}, c_{4}, c_{5}, c_{6}\right\}
$$

that contains the model parameters which are common to all subjects, and $S_{i}=\left\{\theta_{i}, I_{i}, \tau_{i}, \log \gamma_{i}\right\}$, that ${ }_{136}$ contains the parameters which are specific to the $i$-th subject. If we study a dataset including series ${ }_{137}$ from $m$ subjects, then the complete set of parameters is $\boldsymbol{A}=\bigcup_{i=1}^{m} S_{i} \bigcup C$, that contains $n=6+4 m \quad{ }_{138}$ parameters to be estimated. Besides, we use the shorthand $x \sim M H_{G}\left(\sigma_{1}^{2}, J, x_{0}\right)$ to indicate that the ${ }_{139}$ sample $x$ is generated by running a Metropolis-Hastings algorithm with a Gaussian kernel (of variance $\sigma_{1}^{2}$ ) ${ }_{140}$ that produces a Markov chain of J elements with initial condition $x_{0}$. In our case, $x$ may refer to $\tau_{i}$ or ${ }^{141}$ $\log \gamma_{i}$. Complete details are given in Appendix B. ${ }_{142}$

We use an argument-wise notation for probability density functions (pdf's) and probability mass ${ }_{143}$ functions (pmf's). If $x$ and $y$ are two continuous random variables (r.v.), then $p(x)$ and $p(y)$ denote the ${ }_{144}$ pdf's of $x$ and $y$, respectively. These pdf's are possibly different. Similarly, $p(x, y)$ denotes the joint pdf of ${ }_{145}$ $x$ and $y$ and $p(x \mid y)$ is the conditional pd of $x$ given $y$. The same notation is used for pmf's, e.g., if $z$ is a ${ }_{146}$ discrete r.v. then $p(z)$ is its pmf. In our model the only discrete r.v.'s are the indicators $I_{i}, i=1, \ldots, m$. ${ }^{147}$

The proposed MwG algorithm can now be outlined as follows.

Initialisation. For each parameter in $C$, draw an initial sample from its a priori pdf, i.e., draw $\mu_{\theta}^{(0)} \sim{ }^{149}$ $\mathcal{N}(2.75,1),\left(\sigma_{\theta}^{2}\right)^{(0)} \sim I G(2.04,0.065),\left(\sigma^{2}\right)^{(0)} \sim I G(2.05,0.1), \mu_{\gamma}^{(0)} \sim \mathcal{N}(1.1,0.1),\left(\sigma_{\gamma}^{2}\right)^{(0)} \sim \operatorname{IG}(2.2,0.12) \quad{ }_{150}$ and $\pi^{(0)} \sim \operatorname{Beta}(42.5,7.5)$. For each subject $i=1, \ldots, m$ and each parameter in $S_{i}$, draw an initial ${ }_{151}$ 
sample from the corresponding prior pdf/pmf, i.e., draw $\theta_{i}^{(0)} \sim \mathcal{N}\left(\mu_{\theta}^{(0)},\left(\sigma_{\theta}^{2}\right)^{(0)}\right), I_{i}^{(0)} \sim \operatorname{Bernoulli}\left(\pi^{(0)}\right), \quad{ }_{152}$ $\tau_{i}^{(0)} \sim \mathcal{T} \mathcal{N}\left(d_{i}-2,0.75^{2}, d_{i}-5, d_{i}\right)$ and $\log \gamma_{i}^{(0)} \sim \mathcal{N}\left(\mu_{\gamma}^{(0)},\left(\sigma_{\gamma}^{2}\right)^{(0)}\right)$.

Iteration. For $k=1,2, \ldots, K$;

- Draw new samples for the common parameters

$$
c_{j}^{(k)} \sim p\left(c_{j} \mid c_{1}^{(k)}, \ldots, c_{j-1}^{(k)}, c_{j}^{(k)}, c_{j+1}^{(k-1)}, \ldots, c_{6}^{(k-1)}, Y\right), \quad j=1, \ldots, 6,
$$

where $Y$ denotes the full dataset. These conditional pdf's are given explicitly in Appendix A.

- Draw new samples from the subject specific parameters: for $i=1, \ldots, m$

$$
\begin{aligned}
\theta_{i}^{(k)} & \sim p\left(\theta_{i} \mid c_{1}^{(k)}, \ldots, c_{6}^{(k)}, I_{i}^{(k-1)}, \tau_{i}^{(k-1)}, \log \gamma_{i}^{(k-1)}, Y\right) \\
I_{i}^{(k)} & \sim p\left(I_{i} \mid c_{1}^{(k)}, \ldots, c_{6}^{(k)}, \theta_{i}^{(k)}, \tau_{i}^{(k-1)}, \log \gamma_{i}^{(k-1)}, Y\right) \\
\tau_{i}^{(k)} & \sim M H\left(0.02,200, \tau_{i}^{(k-1)}\right) \\
\log \gamma_{i}^{(k)} & \sim M H\left(0.1,200, \log \gamma_{i}^{(k-1)}\right) .
\end{aligned}
$$

The conditional pdf's for $\theta_{i}, I_{i}, \tau_{i}$ and $\log \gamma_{i}$ are given explicitly in Appendix A as well. Running ${ }_{158}$ the MH algorithm to generate the new samples $\tau_{i}^{(k)}$ and $\log \gamma_{i}^{(k)}$ obviously involves the data and the ${ }_{159}$ remaining parameters generated at the $k$-th iteration. Other alternatives for the efficient implementation 160 of Gibbs-based samplers have been explored in the literature [34 40 and can also be applied within the ${ }_{161}$ proposed framework.

\subsection{Change-point detection}

The MwG algorithm is run to generate a Markov chain of $K=10000$ samples for each unknown parameter ${ }_{164}$ in the set $\boldsymbol{A}$. We allow a burn-in period 23 of $L=5000$ samples, which are discarded. The remaining ${ }_{165}$ $K-L=5000$ samples in the Markov chain can be used for estimation.

The estimate of the probability $p\left(I_{i}=1 \mid Y\right)$ is used to detect the presence of a change-point in the time $\quad{ }_{167}$ series of patient $i$. We can estimate $p\left(I_{i}=1 \mid Y\right) \approx \hat{p}\left(I_{i}=1 \mid Y\right)=\frac{1}{K-L} \sum_{j=L+1}^{K} I_{i}^{(j)}$. If $\hat{p}\left(I_{i}=1 \mid Y\right)>0.5 \quad{ }_{168}$ we detect a change-point for the time series of the $i$-th subject. We can estimate the position (time ${ }_{169}$ instant) of this change-point as $\hat{\tau}_{i}=\frac{1}{K-L} \sum_{j=L+1}^{K} \tau_{i}^{(j)}$. Note that this estimate is only meaningful when $\quad{ }_{170}$ 
$\hat{p}\left(I_{i}=1 \mid Y\right)>0.5$. We may compute $\hat{\tau}_{i}$ when $\hat{p}\left(I_{i}=1 \mid Y\right)<0.5$ as well, but in this case we do not detect ${ }_{171}$

a change-point so this value of $\hat{\tau}_{i}$ is not relevant.

It is also of interest to determine whether the change-points for different biomarkers obtained from ${ }_{173}$ the same patient can be expected, a priori, to occur at similar time instants. (Note that biomarker ${ }_{174}$ change-points are not guaranteed to exist for all biomarkers in all patients). We have analysed the available ${ }_{175}$ data in terms of the interval between measurements where the change-point is detected for the different ${ }_{176}$ biomarkers and patients in the dataset.

Let $t_{i, 0}, \ldots, t_{i, k_{i}}$ be the times at which measurements are collected for patient $i$. For a given biomarker, ${ }_{178}$ we say that the change-point is detected at interval 0 when our estimate $\hat{\tau}_{i}$ is less than $t_{i, 0}$; we say that ${ }_{179}$ the change-point is detected at interval 1 when our estimate $\hat{\tau}_{i}$ is between $t_{i, 0}$ and $t_{i, 1}$, etc.. In general, a 180 change-point for subject $i$ is detected at the $j-t h$ interval when $\hat{p}\left(I_{i}=1 \mid Y\right)>0.5$ and $t_{i(j-1)} \leq \hat{\tau}_{i}<t_{i j}, \quad{ }_{181}$ for $j=1, \ldots, k_{i}$. Thus, with $k_{i}$ measurements, the change-point for the given biomarker can be detected ${ }_{182}$ within $k_{i}$ different time slots, labeled $0,1,2, \ldots, k_{i}-1$.

Once the change-points for the available biomarkers have been assigned a time slot each, it is possible to ${ }_{184}$ compare them in pairs and see whether the change-points occur in the same slot or whether one biomarker ${ }_{185}$ precedes the other. As this comparison is carried out for all patients and for each pair of biomarkers, ${ }_{186}$ it is possible to estimate the probability of coincidences (i.e., how likely it is that change-points of two ${ }_{187}$ biomarkers are detected in the same slot) as well as the probability of the change-point of one biomarker ${ }_{188}$ being detected in an earlier slot (e.g., slot 1 for the first biomarker versus slot 2 for the second biomarker). ${ }_{189}$ These summary statistics are easy to compute, robust to change-point estimation errors and provide useful 190 information to assess the diagnosis. Our numerical results are presented and discussed in the following ${ }_{191}$ section.

\section{Results and Discussion}

We have first studied the outcome of applying the proposed method for change-point detection to a control 194 dataset containing time series of 179 healthy subjects and the 6 biomarkers of interest. Each series consists ${ }_{195}$ of 4 or 5 sequential samples available for each biomarker. As expected, the probability that a change-point 196 occurs for the control group is small, less than $2.5 \%$ for all biomarkers, as shown in Table 1). 197

Next we discuss the results for the dataset of patients with ovarian cancer described in Section 2.1 . $\quad 198$ 
Table 1. Change-point detection probabilities for the control dataset.

\begin{tabular}{|c|c|}
\hline Biomarkers & detection probability \\
\hline CA125 & 0.011 \\
\hline HE4 & 0.022 \\
\hline Glycodelin & 0.017 \\
\hline MSLN & 0.011 \\
\hline MMP7 & 0 \\
\hline CYFRA_21-1 & 0.022 \\
\hline
\end{tabular}

CA125, HE4, MMP7, CYFRA 21-1, glycodelin and MSLN were assayed on 2 to 5 serial samples collected ${ }_{199}$ up to 2.5 years prior to diagnosis of iEOC from 44 UKCTOCS patients. As CA125 is the only biomarker 200 currently used in ovarian cancer screening, we investigate here whether taking into account additional 201 biomarkers can improve detection. Table 2 shows the probability that a change-point occurs (at any time ${ }^{202}$ slot) for the different biomarkers. We can see that for CA125, HE4 and Glycodelin the probability is ${ }_{203}$ relatively high (more than 84\%) whereas for MSLN, MMP7 and CYFRA_21-1 this probability is quite ${ }^{204}$ low, specially for the last (20\%). This suggests that while the latter biomarkers do not provide significant ${ }_{205}$ information for cancer detection, the former could play a significant role. We therefore focus on the first 206 three biomarkers, namely CA125, HE4 and Glycodelin in the rest of our study.

Table 2. Change-point detection probabilities for patients.

\begin{tabular}{|c|c|}
\hline Biomarkers & detection probability \\
\hline CA125 & 0.89 \\
\hline HE4 & 0.84 \\
\hline Glycodelin & 0.84 \\
\hline MSLN & 0.50 \\
\hline MMP7 & 0.41 \\
\hline CYFRA_21-1 & 0.20 \\
\hline
\end{tabular}

A change-point was not detected with CA125 in $11 \%$ of patients (and we refer to these cases as false ${ }^{208}$ negatives). Therefore HE4 and/or Glycodelin data was analysed to determine whether they could be used ${ }_{209}$ to improve detection. We detected a change-point in the HE4 data in $80 \%$ of the CA125 false negatives ${ }_{210}$ and in the Glycodelin data in $60 \%$ of the CA125 false negatives. This suggests that use of two biomarkers ${ }^{211}$ together, CA125 plus HE4 or CA125 plus Glycodelin, instead of CA125 alone, can reduce the number of ${ }_{212}$ false negatives from $11 \%$ to $2 \%$ or $4 \%$, respectively. ${ }^{213}$

We then analysed the data from women for whom a change-point was observed in CA125 to determine ${ }_{214}$ 
whether the HE4 and/or Glycodelin data also showed a change-point and, if so, whether there was ${ }_{215}$ coincidence and precedence of the change-points in the same interval of time. Table 3 shows the ${ }_{216}$ numerically estimated probabilities of coincidence and precedence of the change-points in these intervals ${ }^{217}$ by comparing all combinations of pairs for these three biomarkers. Coincidence of time slots was the most ${ }_{218}$ likely event for all pairs, but where there was no coincidence, the change-point of HE4 and Glycodelin ${ }_{219}$ compared to CA125 had a higher probability ( 0.30 versus 0.06 when comparing CA125-HE4 and 0.44 versus ${ }_{220}$ 0.03 when comparing CA125-Glycodelin) of occurring earlier. Thus, another advantage of combining two ${ }_{221}$ biomarkers (CA125 plus HE4 or Glycodelin) instead of CA125 alone is the potential for earlier detection ${ }_{222}$ of the disease.

Table 3. Probabilities of coincidence and precedence of change-points.

\begin{tabular}{|c|c|c|c|}
\hline Pairs of biomarkers & coincidence & first earlier & first later \\
\hline CA125 - HE4 & 0.64 & 0.06 & 0.30 \\
\hline CA125 - Glycodelin & 0.53 & 0.03 & 0.44 \\
\hline HE4 - Glycodelin & 0.74 & 0.06 & 0.21 \\
\hline
\end{tabular}

In order to corroborate these results, we have plotted histograms related to the change-point estimates ${ }^{224}$ obtained with the CA125, HE4 and Glycodelin data, respectively, for every woman. To be specific, 225 let $\hat{\tau}_{C A 125}^{(i)}, \hat{\tau}_{H E 4}^{(i)}$ and $\hat{\tau}_{\text {Glycodelin }}^{(i)}$ be the change-point estimates, measured in years, obtained from the ${ }^{226}$ corresponding time series data for the $i$-th patient (and, as there are 44 individuals in the dataset, ${ }_{227}$ $i=1,2, \ldots, 44)$. Fig. 2 shows the histograms for the differences between all three possible pairs of ${ }^{228}$ estimates:

- $\operatorname{plot}(\mathrm{a})$ displays the histogram for the CA125-HE4 differences, i.e., $\hat{\tau}_{C A 125}^{(i)}-\hat{\tau}_{H E 4}^{(i)}$;

- plot (b) displays the histogram for the CA125-Glycodelin differences, i.e., $\hat{\tau}_{C A 125}^{(i)}-\hat{\tau}_{\text {Glycodelin }}^{(i)}$; and ${ }^{231}$

- plot (c) displays the histogram for the HE4-Glycodelin differences, i.e., $\hat{\tau}_{H E 4}^{(i)}-\hat{\tau}_{\text {Glycodelin }}^{(i)}$.

Note that these differences can only be computed for those patients for whom a change-point has been ${ }_{233}^{233}$ detected for the two biomarkers in the pair (e.g., the histogram in Fig. 2(a) includes the time differences ${ }^{234}$ for individuals for whom a change-point was detected both in the CA125 data and in the HE4 data). ${ }_{235}$ We can observe that all histograms are approximately centered around zero, since all biomarkers tend ${ }_{236}$ to estimate the change-point in the same time interval with high probability, but a closer look actually ${ }_{237}$ shows that the histograms of Figs. 2 (a)-(b) are slightly shifted to the right, which is consistent with with ${ }^{238}$ 
the observation in Table 3 that, when there is no coincidence in the same time slot, the change-points ${ }_{239}^{239}$ detected in the HE4 and Glycodelin data appear earlier than the change-point detected in CA125 data 240 with high probability.
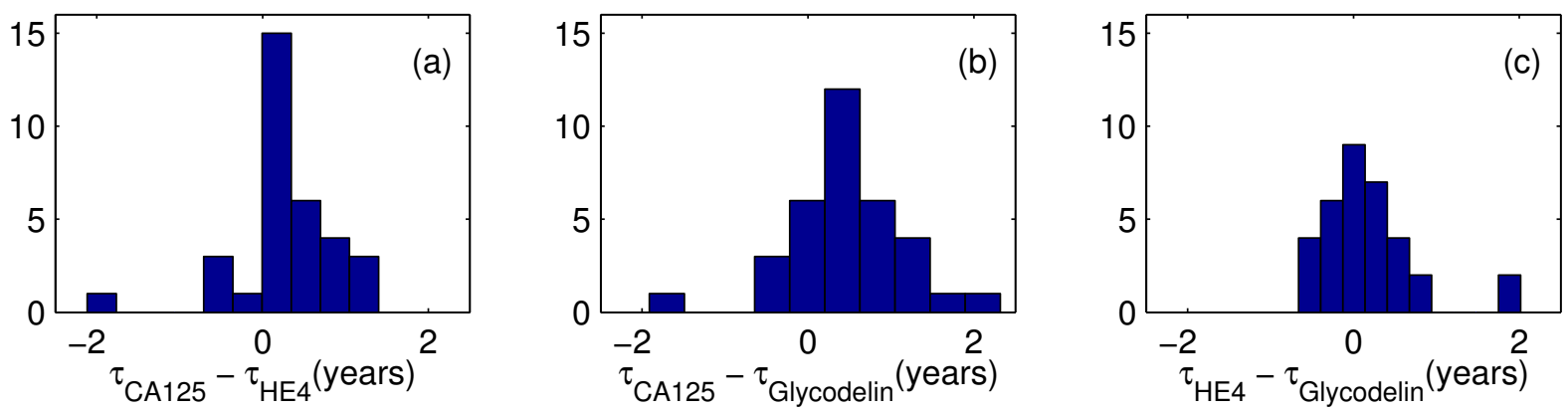

Fig 2. Histograms for the differences in time measured in years between change-point estimates for the following pairs of biomarkers: (a) CA125-HE4, (b) CA125-Glycodelin, (c) HE4-Glycodelin.

\section{Conclusions}

We have designed a Bayesian change-point (BCP) model, based on the approach of Skates et al. [5], that may be used in the analysis of biomarker time-series data, as an aid to the diagnosis of ovarian cancer. It is known that, in approximately $85 \%$ of women who go on to be diagnosed with ovarian cancer, CA125 data display a change-point (turning from stationary to a linearly increasing trend in the transformed measurements) prior to diagnosis. The detection of such change-points has been used to diagnose the disease earlier. The contribution of this paper is twofold:

- For the proposed BCP model we have explicitly computed the conditional probability distributions of all the model parameters. The availability of the conditional probability distribution of all model parameters may have wide theoretical and practical advantages. In particular, it enables the design of efficient and stable computational estimation algorithms for these parameters and, specially, for the change-points.

- In a real time-series dataset of samples preceding diagnosis of ovarian cancer, we have applied the ${ }^{254}$ proposed BCP model and a Gibbs-sampling based estimation algorithm to detect and estimate 255 change-points in the tumour markers CA125, HE4, MMP7, CYFRA_21-1, MSLN and Glycodelin. In 256 
this dataset, diagnosis based on CA125 alone yields $\approx 11 \%$ false negatives, i.e., diagnosed patients ${ }^{257}$ for whom the CA125 data does not display a change-point. We have shown that by jointly analysing 258 the CA125 and Glycodelin data, the rate of false negatives is reduced to 4\%, while the analysis of ${ }_{259}$ CA125 together with HE4 further reduces this rate to a mere $2 \%$.

Our results suggest that the change-point approach with combined assay of HE4 or Glycodelin along with 261 CA125 can lead to an earlier diagnosis compared to an approach based on CA125 alone. In particular, we ${ }_{262}$ found that for those patients for whom change-points are detected both with CA125 and HE4/Glycodelin, 263 the change-point occurs earlier in the HE4/Glycodelin data in a significant proportion of cases. 264

The proposed Bayesian detection and estimation scheme admits several extensions that can be 265 implemented with limited additional computations. In particular, given the approximate posterior 266 distribution produced by the MwG sampler, it is possible to assess the credibility of the detected change- ${ }^{267}$ points as well as the accuracy of the estimated times where the change takes place. This capability may 268 lead to further improvements in the reliability of the change-point detection scheme.

\section{A Appendix: Conditional distributions}

The conditional distribution for each parameter in the proposed Bayesian hierarchical model can ${ }^{271}$ be found analytically. Let $\boldsymbol{A}=\bigcup_{i=1}^{m} S_{i} \cup C$ represent the complete set of parameters, where $C={ }^{272}$ $\left\{\mu_{\theta}, \mu_{\gamma}, \sigma_{\theta}^{2}, \sigma_{\gamma}^{2}, \sigma^{2}, \pi\right\}$ denotes the parameters common to every subject, $S_{i}=\left\{\theta_{i}, I_{i}, \tau_{i}, \log \gamma_{i}\right\}$, contains ${ }^{273}$ the parameters which are specific to the $i$-th subject and $m$ represents the number of subjects. Notice ${ }^{274}$ that $6+4 m$ is the number of parameters to be estimated. 275 
The full conditional distributions for the common parameters are:

$$
\begin{aligned}
& p\left(\mu_{\theta} \mid \boldsymbol{A} \backslash\left\{\mu_{\theta}\right\}, Y\right)=\mathcal{N}\left(\hat{\mathrm{m}}_{0}, \hat{v}^{2}\right) \quad \text { where }\left\{\begin{array}{l}
\hat{\mathrm{m}}_{0}=\frac{\mathrm{m}_{0} \sigma_{\theta}^{2}+v^{2} \sum_{i=1}^{m} \theta_{i}}{m v^{2}+\sigma_{\theta}^{2}} \\
\hat{v}^{2}=\frac{v^{2} \sigma_{\theta}^{2}}{m v^{2}+\sigma_{\theta}^{2}}
\end{array}\right. \\
& p\left(\mu_{\gamma} \mid \boldsymbol{A} \backslash\left\{\mu_{\gamma}\right\}, Y\right)=\mathcal{N}\left(\hat{\mathrm{m}}_{0}, \hat{v}^{2}\right) \quad \text { where }\left\{\begin{array}{l}
\hat{\mathrm{m}}_{0}=\frac{\mathrm{m}_{0} \sigma_{\gamma}^{2}+v^{2} \sum_{i=1}^{m} \log \gamma_{i}}{m v^{2}+\sigma_{\gamma}^{2}} \\
\hat{v}^{2}=\frac{v^{2} \sigma_{\gamma}^{2}}{m v^{2}+\sigma_{\gamma}^{2}}
\end{array}\right. \\
& p\left(\sigma_{\theta}^{2} \mid \boldsymbol{A} \backslash\left\{\sigma_{\theta}^{2}\right\}, Y\right)=I G(\hat{\alpha}, \hat{\beta}) \quad \text { where }\left\{\begin{array}{l}
\hat{\alpha}=\alpha+m / 2 \\
\hat{\beta}=\beta+\frac{1}{2} \sum_{i=1}^{m}\left(\theta_{i}-\mu_{\theta}\right)^{2}
\end{array}\right. \\
& p\left(\sigma_{\gamma}^{2} \mid \boldsymbol{A} \backslash\left\{\sigma_{\gamma}^{2}\right\}, Y\right)=I G(\hat{\alpha}, \hat{\beta}) \quad \text { where }\left\{\begin{array}{l}
\hat{\alpha}=\alpha+m / 2 \\
\hat{\beta}=\beta+\frac{1}{2} \sum_{i=1}^{m}\left(\log \gamma_{i}-\mu_{\gamma}\right)^{2}
\end{array}\right. \\
& p\left(\sigma^{2} \mid \boldsymbol{A} \backslash\left\{\sigma^{2}\right\}, Y\right)=I G(\hat{\alpha}, \hat{\beta}) \quad \text { where }\left\{\begin{array}{l}
\hat{\alpha}=\alpha+\frac{1}{2} \sum_{i=1}^{m} k_{i} \\
\hat{\beta}=\beta+\frac{1}{2} \sum_{i=1}^{m}\left(\sum_{j=1}^{k_{i}}\left(Y_{i j}-E_{i j}\right)^{2}\right) \\
\text { being } E_{i j}=\theta_{i}+I_{i} \gamma_{i}\left(t_{i j}-\tau_{i}\right)^{+}
\end{array}\right. \\
& p(\pi \mid \boldsymbol{A} \backslash\{\pi\}, Y)=\operatorname{Beta}(\hat{a}, \hat{b}) \quad \text { where }\left\{\begin{array}{l}
\hat{a}=a+\sum_{i=1}^{m} I_{i} \\
\hat{b}=b+m-\sum_{i=1}^{m} I_{i}
\end{array}\right.
\end{aligned}
$$

where $k_{i}$ is the number of observations for the $i$-th patient, $\mathrm{m}_{0}$ and $v^{2}$ generically represent the mean ${ }_{277}$ and the variance, respectively, of the prior Gaussian distributions of the corresponding parameters (in an ${ }_{278}$ abuse of notation, since these prior means and variances can be different for different parameters) and ${ }^{279}$ $\hat{\mathrm{m}}_{0}$ and $\hat{v}^{2}$ represent the same magnitudes for the posterior Gaussian distributions of the corresponding 280 parameters. The parameters $\alpha$ and $\beta$ are used for the prior inverse gamma distribution, $I G(\alpha, \beta)$, which ${ }^{281}$ has mean $\beta /(\alpha-1)$ and variance $\beta^{2} /\left[(\alpha-1)^{2}(\alpha-2)\right]$, while we denote as $\hat{\alpha}$ and $\hat{\beta}$ the parameters of the ${ }^{282}$ conjugate posterior inverse gamma distribution, $I G(\hat{\alpha}, \hat{\beta})$. We have $I G(\cdot, \cdot)$ priors and posteriors for the ${ }^{283}$ variance parameters $\sigma_{\theta}^{2}, \sigma_{\gamma}^{2}$ and $\sigma^{2}$. For each one of these parameters, the prior parameters $\alpha$ and $\beta$ can ${ }^{284}$ be different. Similarly, we use $a$ and $b$ for the prior Beta distribution of the model parameter $\pi$, and $\hat{a}{ }^{285}$ and $\hat{b}$ for the parameters of the corresponding posterior Beta function. 
The full conditional distributions for the model parameters which are specific to each subject are

$$
\begin{aligned}
& p\left(\theta_{i} \mid \boldsymbol{A} \backslash\left\{\theta_{i}\right\}, Y\right)=\mathcal{N}\left(\hat{\mathbf{m}}_{i}, \hat{v}_{i}^{2}\right), \quad \text { where }\left\{\begin{array}{l}
\hat{\mathbf{m}}_{i}=\frac{\mu_{\theta} \sigma^{2}+\sigma_{\theta}^{2} \sum_{j=1}^{k_{i}} H_{i j}}{k_{i} \sigma_{\theta}^{2}+\sigma^{2}} \\
\hat{v}_{i}^{2}=\frac{\sigma^{2} \sigma_{\theta}^{2}}{k_{i} \sigma_{\theta}^{2}+\sigma^{2}} \text { being } \\
H_{i j}=Y_{i j}-I_{i} \gamma_{i}\left(t_{i j}-\tau_{i}\right)^{+}
\end{array}\right. \\
& p\left(I_{i} \mid \boldsymbol{A} \backslash\left\{I_{i}\right\}, Y\right) \quad=\quad \operatorname{Bern}(1, \hat{\pi}), \quad \text { where }\left\{\begin{array}{l}
\hat{\pi}=\frac{\pi \prod_{j=1}^{k_{i}} \mathcal{N}\left(Y_{i j} ; E_{i j}, \sigma^{2}\right)}{\pi \prod_{j=1}^{k_{i}} \mathcal{N}\left(Y_{i j} ; E_{i j}, \sigma^{2}\right)+(1-\pi) \prod_{j=1}^{k_{i}} \mathcal{N}\left(Y_{i j} ; \theta_{i}, \sigma^{2}\right)} \\
\text { being } E_{i j}=\theta_{i}+\gamma_{i}\left(t_{i j}-\tau_{i}\right)^{+}
\end{array}\right. \\
& p\left(\tau_{i} \mid \boldsymbol{A} \backslash\left\{\tau_{i}\right\}, Y\right) \quad \propto \quad \prod_{j=1}^{k_{i}} \mathcal{N}\left(Y_{i j} ; E_{i j}, \sigma^{2}\right) \mathcal{T} \mathcal{N}\left(\tau_{i} ; d_{i}-2,0.75, d_{i}-5, d_{i}\right), \\
& \text { where } \quad E_{i j}=\theta_{i}+I_{i} \gamma_{i}\left(t_{i j}-\tau_{i}\right)^{+} \\
& p\left(\log \gamma_{i} \mid \boldsymbol{A} \backslash\left\{\log \gamma_{i}\right\}, Y\right) \quad \propto \quad \prod_{j=1}^{k_{i}} \mathcal{N}\left(Y_{i j} ; E_{i j}, \sigma^{2}\right) \mathcal{N}\left(\log \gamma_{i} ; \mu_{\gamma}, \sigma_{\gamma}^{2}\right), \\
& \text { where } \quad E_{i j}=\theta_{i}+I_{i} \gamma_{i}\left(t_{i j}-\tau_{i}\right)^{+} .
\end{aligned}
$$

where $i$ represents the subject, $\hat{\mathrm{m}}_{i}$ and $\hat{v}_{i}^{2}$ represent, respectively, the mean and the variance of the ${ }^{288}$ posterior Gaussian distributions for the parameter $\theta_{i}, \pi$ and $\hat{\pi}$ are, respectively, the success probability of ${ }_{289}$ the prior and posterior Bernoullli distributions and $\operatorname{Bern}(1, \hat{\pi})$ represents a Bernoulli distribution with 290 success probability $\hat{\pi}$.

It is possible seek alternative implementations of the Gibbs sampler by identifying functions proportional $\quad 292$ to the full conditionals in the factors of the joint pdf of the parameters and the data. Again, let $\boldsymbol{A}$ denote ${ }^{293}$ the set of all model parameters and let $Y$ denote the set of all observations (for all time instants and all ${ }^{294}$ patients). Then, the joint pdf can be written as

$$
p(\boldsymbol{A}, Y)=p(Y \mid \boldsymbol{A}) p(\boldsymbol{A})
$$

where $p(Y \mid \boldsymbol{A})$ is the likelihood function and $p(\boldsymbol{A})$ is the joint prior pdf of the parameters. The latter is ${ }_{296}$ readily deduced from the model structure, namely

$$
p(\boldsymbol{A})=\left[\prod_{i=1}^{m} p\left(I_{i} \mid \pi\right) p\left(\theta_{i} \mid \mu_{\theta}, \sigma_{\theta}^{2}\right) p\left(\log \gamma_{i} \mid \mu_{\gamma}, \sigma_{\gamma}^{2}\right) p\left(\tau_{i}\right)\right] p\left(\sigma^{2}\right) p\left(\mu_{\theta}\right) p\left(\sigma_{\theta}^{2}\right) p(\pi) p\left(\mu_{\gamma}\right) p\left(\sigma_{\gamma}^{2}\right)
$$

where $p\left(\sigma^{2}\right)=I G(2.05,0.1), p\left(\mu_{\theta}\right)=\mathcal{N}(2.75,1), p\left(\sigma_{\theta}^{2}\right)=I G(2.04,0.065), p(\pi)=\operatorname{Beta}(42.5,7.5), \quad{ }^{298}$ 
$p\left(\mu_{\gamma}\right)=\mathcal{N}(1.1,0.1)$ and $p\left(\sigma_{\gamma}^{2}\right)=I G(2.2,0.12)$ are the prior densities of the common parameters of ${ }^{299}$ the model and $p\left(I_{i} \mid \pi\right)=\operatorname{Bern}(1, \pi), p\left(\theta_{i} \mid \mu_{\theta}, \sigma_{\theta}^{2}\right)=\mathcal{N}\left(\mu_{\theta}, \sigma_{\theta}^{2}\right), p\left(\log \gamma_{i} \mid \mu_{\gamma}, \sigma_{\gamma}^{2}\right)=\mathcal{N}\left(\mu_{\gamma}, \sigma_{\gamma}^{2}\right)$ and $p\left(\tau_{i}\right)=\quad 300$ $\mathcal{T} \mathcal{N}\left(d_{i}-2,0.75, d_{i}-5, d_{i}\right)$ are the priors of the subject-specific parameters.

The likelihood function can be obtained in closed form using the total probability theorem, for $I_{i}=0 \quad 302$ and $I_{i}=1$, as

$$
\begin{aligned}
p(Y \mid \boldsymbol{A}) & =\prod_{i=1}^{m} \prod_{j=1}^{k_{i}}\left[p\left(Y \mid I_{i}=0, \boldsymbol{A} \backslash\left\{I_{i}\right\}\right) p\left(I_{i}=0 \mid \boldsymbol{A} \backslash\left\{I_{i}\right\}\right)+p\left(Y \mid I_{i}=1, \boldsymbol{A} \backslash\left\{I_{i}\right\}\right) p\left(I_{i}=1 \mid \boldsymbol{A} \backslash\left\{I_{i}\right\}\right)\right] \\
& =\prod_{i=1}^{m} \prod_{j=1}^{k_{i}}\left[(1-\pi) \mathcal{N}\left(Y_{i j} ; \theta_{i}, \sigma^{2}\right)+\pi \mathcal{N}\left(Y_{i j} ; \theta_{i}+\gamma_{i}\left(t_{i j}-\tau_{i}\right)^{+}, \sigma^{2}\right)\right] .
\end{aligned}
$$

The joint pdf is obtained by substituting (8) and (9) back into (7). The resulting function is proportional 304 to the full conditional of a given model parameter if we assign a fixed value to all other parameters. 305

\section{B Appendix: Metropolis-Hastings algorithm}

The Metropolis-Hastings algorithm can be used to draw samples from any pdf $p(x)$, provided the value 307 of a function $f(x) \propto p(x)$ can be computed. It works by generating a Markov chain which has $p(x)$ as 308 a stationary (limiting) distribution [23]. The sample $x_{t+1}$ in the chain is produced using a proposal 309 conditional pdf $g\left(x_{t+1} \mid x_{t}\right)$ followed by a random acceptance test. The Metropolis algorithm is a special 310 case of the Metropolis-Hastings algorithm where the proposal function is symmetric and it can be outlined 311 as follows.

Initialisation Choose an arbitrary point $x_{0}$ to be the first sample, and choose an arbitrary symmetric 313 proposal pdf $g(x \mid y)(g(x \mid y)=g(y \mid x))$. In this work we have considered a Gaussian distribution centered 314 at $y$.

Iteration. For $t=1,2, \ldots$ :

- Draw a candidate $x^{\prime}$ from the proposal pdf $g\left(x^{\prime} \mid x_{t-1}\right)$.

- Compute the acceptance ratio $\alpha=f\left(x^{\prime}\right) / f\left(x_{t-1}\right)$.

- Draw a uniform sample $u \sim U(0,1)$. If $u<\alpha$ then accept $x^{\prime}$ and set $x_{t}=x^{\prime}$. Otherwise, set 319 $x_{t}=x_{t-1}$ 
In particular, in our work for each iteration in the Gibbs sampler (see Section 3.1) in order to draw 321 from the conditional distributions of parameters $\tau_{i}$ and $\log \gamma_{i}$ for each patient $i$ we run a Metropolis ${ }_{322}$ algorithm with 200 steps for each of these parameters. The proposal density has been taken Gaussian, 323 centred in the previous value of the corresponding parameter, where the variance for $\tau_{i}, i=1, \ldots, m \quad 324$ is 0.02 and the variance for $\log \gamma_{i}, i=1, \ldots, m$ is 0.1 . The functions $f\left(\tau_{i}\right) \propto p\left(\tau_{i} \mid \boldsymbol{A} \backslash\left\{\tau_{i}\right\}, Y\right)$ and 325 $f\left(\log \gamma_{i}\right) \propto p\left(\log \gamma_{i} \mid \boldsymbol{A} \backslash\left\{\log \gamma_{i}\right\}, Y\right)$ are given in Appendix A.

\section{Acknowledgments}

This research was funded by Cancer Research UK and the Eve Appeal Gynaecological Cancer Research 328 Fund (grant ref. A12677) and was supported by the National Institute for Health Research (NIHR) ${ }_{329}$ University College London Hospitals (UCLH) Biomedical Research Centre. UKCTOCS was core funded 330 by the Medical Research Council, Cancer Research UK, and the Department of Health with additional 331 support from the Eve Appeal, Special Trustees of Bart's and the London, and Special Trustees of UCLH. 332 I. P. M. acknowledges the financial support of the Spanish Ministry of Economy and Competitiveness 333 (projects TEC2012-38883-C02-01, TEC2015-69868-C2-1-R and FIS2013-40653-P) and financial support 334 from the Spanish Ministry of Education, Culture and Sport (Mobility Award PRX15/00378). 335

\section{References}

1. http://cancerresearchuk.org

2. P.O. Brown, C. Palmer, The preclinical natural history of serous ovarian cancer: defining the target for early detection, PLoS Med. 6 (2009) e1000114.

3. S.S. Hori, S.S. Gambhir, Mathematical model identifies blood biomarker-based early cancer detection strategies and limitations, Sci. Transl. Med. 3 (2011) 109ra116.

4. J.A. Rauh-Hain, T.C. Krivak, M.G. Del Carmen, A.B. Olawaiye, Ovarian cancer screening and early detection in the general population, Reviews in obstetrics and gynecology 4 (2011), 15-21, PubMed PMID: 21629494. Pubmed Central PMCID: 3100094. 
5. S.J. Skates, D.K. Pauler, and I.J. Jacobs, Screening based on the risk of cancer calculation from Bayesian hierarchical change-point and mixture models of longitudinal markers, J. Am. Stat. Assoc. 96 (2001) 429-439.

6. S.J. Skates, F.J. Xu, Y.H. Yu, K. Sjovall, N. Einhorn, Y. Chang, R.C. Bast Jr., R.C. Knapp, Toward an optimal algorithm for ovarian cancer screening with longitudinal tumor markers, Cancer 76 (1995) 2004-2010.

7. S.J. Skates, U. Menon, N. MacDonald, A.N. Rosenthal, D.H. Oram, R.C. Knapp, I.J. Jacobs, Calculation of the risk of ovarian cancer from serial CA-125 values for preclinical detection in postmenopausal women, J. Clin. Oncol. 21 (2003) 206-211.

8. T. Van Gorp, I. Cadron, E. Despierre et al., HE4 and CA125 as a diagnostic test in ovarian cancer: prospective validation of the Risk of Ovarian Malignancy Algorithm, British Journal of Cancer 104 (2011) 863-870.

9. N. Ghasemi, S. Ghobadzadeh, M. Zahraei et al., HE4 combined with CA125: favorable screening tool for ovarian cancer, Medical Oncology 31(2014), article 808.

10. R.G. Moore, D.S. McMeekin, A.K. Brown, P. DiSilvestro, M.C. Miller et al., A novel multiple marker bioassay utilizing HE4 and CA125 for the prediction of ovarian cancer in patients with a pelvic mass, Gynecol. Oncol. 112 (2009) 40-46.

11. G.L. Anderson, M. McIntosh, L. Wu et al., Assessing lead time of selected ovarian cancer biomarkers: a nested case-control study, Journal of the National Cancer Institute 102 (2010) 26-38.

12. R.G. Moore, A.K. Brown, M.C. Miller, S. Skates, W.J. Allard, T. Verch, M. Steinhoff, G. Messerlian, P. DiSilvestro, C.O. Granai, R.C. Bast Jr, The use of multiple novel tumor biomarkers for the detection of ovarian carcinoma in patients with a pelvic mass, Gynecol. Oncol. 108 (2008) 402-8.

13. L.J. Havrilesky, C.M. Whitehead, J.M. Rubatt et al., Evaluation of biomarker panels for early stage ovarian cancer detection and monitoring for disease recurrence, Gynecol. Oncol. 110 (2008) 374-382.

14. O. Blyuss, A. Gentry-Maharaj, E-O. Fourkala, A. Ryan, A. Zaikin, U. Menon, I. Jacobs, J.F. Timms, Serial patterns of ovarian cancer biomarkers in a prediagnosis longitudinal dataset, BioMed. Research International 2015 (2015) 681416. 
15. U. Hasholzner, L. Baumgartner, P. Stieber, W. Meier, K. Hofmann, A. Fateh-Moghadam, Significance of the tumour markers CA 125 II, CA 72-4, CASA and CYFRA 21-1 in ovarian carcinoma, Anticancer Research 14 (1994) 2743-2746.

16. M.W. McIntosh, C. Drescher, B. Karlan B et al., Combining CA125 and SMR serum markers for diagnosis and early detection of ovarian carcinoma, Gynecol. Oncol. 95 (2004) 9-15.

17. M.A. Karlsen, E.V. Høgdall, I.J. Christensen, C. Borgfeldt, G. Kalapotharakos, L. ZdrazilovaDubska, J. Chovanec, C.A. Lok, A. Stiekema, I. Mutz-Dehbalaie, A.N. Rosenthal, E.K. Moore, B.A. Schodin, W.W. Sumpaico, K. Sundfeldt, B. Kristjansdottir, I. Zapardiel, C.K. Høgdall, A novel diagnostic index combining HE4, CA125 and age may improve triage of women with suspected ovarian cancer - An international multicenter study in women with an ovarian mass, Gynecol. Oncol. 138 (2015) 640-646.

18. M. Montagnana, E. Danese, Ruzzenente O et al.. The ROMA (Risk of Ovarian Malignancy Algorithm) for estimating the risk of epithelial ovarian cancer in women presenting with pelvic mass: is it really useful?, Clinical Chemistry and Laboratory Medicine 49 (2011) 521-525.

19. S.S. Buys, E. Partridge, A. Black A et al., Effect of screening on ovarian cancer mortality: the Prostate, Lung, Colorectal and Ovarian (PLCO) cancer screening randomized controlled trial, The Journal of the American Medical Association 305 (2011) 2295-2302.

20. Cramer DW, Bast RC Jr, Berg CD, Diamandis EP, Godwin AK, Hartge P, Lokshin AE, Lu KH, McIntosh MW, Mor G, Patriotis C, Pinsky PF, Thornquist MD, Scholler N, Skates SJ, Sluss PM, Srivastava S, Ward DC, Zhang Z, Zhu CS, Urban N. Ovarian cancer biomarker performance in prostate, lung, colorectal, and ovarian cancer screening trial specimens. Cancer prevention research 2011;4(3): 365-74.

21. A. Bischof, V. Briese, D-U. Richter, C. Bergemann, K. Friese, U. Jeschke, Measurement of glycodelin A in fluids of benign ovarian cysts, borderline tumours and malignant ovarian cancer, Anticancer Research 25 (2005) 1639-1644.

22. N. Urban, J.D. Thorpe, L.A. Bergan et al., Potential role of HE4 in multimodal screening for epithelial ovarian cancer, Journal of the National Cancer Institute 103 (2011) 1630-1634. 
23. C. Robert, G. Casella, Monte Carlo statistical methods, Springer, 2004.

24. G.O. Roberts, J.S. Rosenthal, Harris recurrence of Metropolis-within-Gibbs and trans-dimensional Markov chains, The Annals of Applied Probability 16 (2006) 2123-2139.

25. UKCTOCS (International Standard Randomised Controlled Trial, number ISRCTN22488978; NCT00058032) was approved by the UK North West Multicentre Research Ethics Committees (North West MREC 00/8/34), 2003, https://clinicaltrials.gov/.

26. FIGO (International Federation of Ginecology and Obstetrics) http://www.figo.org

27. S.E. Kabawat, R.C. Bast, W.R. Welch, R.C. Knapp, R.B. Colvin, Immunopathologic characterisation of a monoclonal antibody that recognises common surface antigens of human ovarian tumours of serous, endometroid, and clear cell types, American Journal of Clinical Pathology 79 (1983) 98-104.

28. S. Campbell, T. Bourne, Ultrasound screening for ovarian cancer. British Medical Journal 70 (1992) $41-55$.

29. N. Einhorn, K. Sjovall, R.C. Knapp, P. Hall, R.E. Scully, R.C. Bast Jr., V.R. Zurawski Jr., Prospective evaluation of serum CA 125 levels for early detection of ovarian cancer. Obstetrics and Gynecology 80 (1992) 14-18.

30. J.R. van Nagell, P.D. DePriest, H.H. Gallion, E.J. Pavlik, Ovarian cancer screening, Cancer 71 (1993) 1523-1528.

31. H. Haario, E. Saksman, J. Tamminen, Componentwise adaptation for high dimensional MCMC, Computational Statistics 20 (2005), 265-273.

32. R.A. Levine, Z. Yu, W.G. Hanley, J.J. Nitao, Implementing componentwise Hastings algorithms, Computational Statistics and Data Analysis 48 (2005) 363-389.

33. A.A. Johnson, G.L. Jones, R.C. Neath, Component-Wise Markov Chain Monte Carlo: Uniform and Geometric Ergodicity under Mixing and Composition Statistical Science, 28 (2013) 360-375.

34. C. Ritter, M.A. Tanner. Facilitating the Gibbs sampler: The Gibbs stopper and the griddy-Gibbs sampler, Journal of the American Statistical Association 87 (1992) 861-868. 
35. L. Martino, H. Yang, D. Luengo, J. Kanniainen, J. Corander, A fast universal self-tuned sampler within Gibbs sampling. Digital Signal Processing 47 (2015) 68-83.

36. W.R. Gilks, N.G. Best, and K.K.C. Tan, Adaptive rejection Metropolis sampling within Gibbs sampling. Applied Statistics 44 (1995) 455-472.

37. R. Meyer, B. Cai, F. Perron, Adaptive rejection Metropolis sampling using Lagrange interpolation polynomials of degree 2. Computational Statistics and Data Analysis 52 (2008) 3408-3423.

38. B. Cai, R. Meyer, F. Perron, Metropolis-Hastings algorithms with adaptive proposals, Statistics and Computing 18 (2008) 421-433.

39. L. Martino, J. Read, D. Luengo, Independent doubly adaptive rejection Metropolis sampling within Gibbs sampling, IEEE Transactions on Signal Processing 63 (2015) 3123- 3138.

40. W. Shao, G. Guo, F. Meng, S. Jia, An efficient proposal distribution for Metropolis- Hastings using a b-splines technique, Computational Statistics and Data Analysis 53 (2013) 465-478.

41. M. Brewer and C. Aitken, Discussion on the meeting on the Gibbs sampler and other Markov Chain Monte Carlo methods, Journal of the Royal Statistical Society, Series B, 55 (1993) 69-70.

42. P. Muller, A generic approach to posterior integration and Gibbs sampling, Technical Report 91-09, Department of Statistics of Purdue University, 1991.

43. C. Fox, A Gibbs sampler for conductivity imaging and other inverse problems, Proc. of SPIE, Image Reconstruction from Incomplete Data VII, 8500 (2012) 1-6. 